\section{A Stable Lead Citrate Stain for Grids}

Leona Cohen-Gould

Weill Medical College of Cornell University, New York, NY

lcgould@med.cornell.edu

For people in a small EM lab, keeping stable stain solutions that don't accumulate precipitates over time can be a problem. Over the last 26 years, I have adapted the method originally described by Venable and Coggeshall (1) to address this need.

\section{Materials:}

Lead Citrate powder

$10 \mathrm{~N} \mathrm{NaOH}$

Deionized or double distilled water

$20 \mathrm{ml}$ syringe with a cap for the tip

PVDF membrane syringe filter with 0.2 micrometer pores

$\sim 0.01 \mathrm{~N} \mathrm{NaOH}$ solution (carbonate-free) for rinsing grids (I make this up by adding 2 pellets of $\mathrm{NaOH}$ to $500 \mathrm{ml}$ deionized water)

\section{Method:}

Heat $15 \mathrm{ml}$ water on a hot plate until bubbles are released, but do not allow it to reach a full boil. Let it stand to cool slightly and retain $10 \mathrm{ml}$.

Weigh out $0.02 \mathrm{~g}$ lead citrate powder.

Add the lead citrate powder to the warm, degassed water and swirl gently to mix, taking care not to agitate the water thus introducing air.

Add one drop (from a $1.5 \mathrm{ml}$ transfer pipet) of $10 \mathrm{~N} \mathrm{NaOH}^{\star}$ and continue to swirl. This will adjust the $\mathrm{pH}$ and aid mixing. Do not worry if all of the lead citrate doesn't dissolve. The original paper (1) used a range of 0.01-0.04 gm/10 ml.

Draw the solution up into the syringe and expel any air. Cap the syringe, wrap it in foil and store at room temperature.

\section{How to Use:}

Prepare a staining chamber by placing a square of Parafilm (American National Can Co.) in the bottom portion of a $100 \mathrm{~mm}$ diameter plastic tissue culture dish. Place approximately 20 pellets of $\mathrm{NaOH}$ in the dish, beside the Parafilm. This will absorb water and carbonates from the air inside the chamber, helping to prevent precipitates.

Remove the syringe from the foil and replace the cap with a filter. Dispense the first drop as waste, then lay out an array of drops on the Parafilm. I usually avoid staining more than 4 sets of grids at a time, to minimize the chances of contamination.

Lay grids, sections down, on the drops of stain. Take care not to breathe on the stain. (I lift the lid as if I was feeding cultured cells, keeping the top of the lid tilted toward me). If you are not staining serial sections, 3 or 4 grids of the same sample can be placed on a single drop. Replace the cover on the chamber.

Stain for 5-6 minutes.

Using the same lid-lifting technique, pick a single grid from a drop of stain and immediately dip repeatedly (about 20 times) into a beaker of the $0.01 \mathrm{~N} \mathrm{NaOH}$. This will wash off the stain while minimizing precipitates. Wash the grid with deionized water, either by dipping or with a gentle stream from a wash bottle and use filter paper to blot between the tips of the forceps and the edge of the grid. Place on a clean piece of filter paper, or hold in the forceps until dry.

This stain may be used after staining with uranyl acetate (UA), or alone if the tissues have received en bloc staining with UA. I have used this technique with much success for well over 20 years.

The formulation of the stain is that described by Venable and Coggeshall in their original article (1). My adaptations are the storage conditions and the initial wash in the dilute $\mathrm{NaOH}$.

\section{References}

Venable, J.H. and Coggeshall, R. A simplified lead citrate stain for use in electron microscopy. J. Cell Biol. 25, 407-408, 1965.

$\left({ }^{*}\right.$ As with Reynold's Lead citrate, excess $\mathrm{NaOH}$ will raise the $\mathrm{pH}$ too much and result in a negative contrast effect).

\section{Microscopy Microanalysis Table of Contents Preview Volume 11, Number 5, October 2005}

SPECIAL IssuE: Frontiers of Electron Microscopy in Materials Science

Introduction Wayne King

Recent Advances in Electron Tomography: TEM and HAADF-STEM

Tomography for Materials Science and Semiconductor Applications Christian Kübel, Andreas Voigt, Remco Schoenmakers, Max Otten, David Su, Tan-Chen Lee, Anna Carlsson, and John Bradley

Structure Determination of Atomically Controlled Crystal Architectures Grown within Single Wall Carbon Nanotubes Angus I. Kirkland, Rüdiger R. Meyer, J. Sloan, and J.L. Hutchinson

TEM Investigation of Nanophase Aluminum Powder Valéry Y. Gertsman and Queenie S.M. Kwok

$\mathrm{Lal}_{2} @(18,3)$ SWNT: The Unprecedented Structure of a Lal 2 "Crystal,"

Encapsulated within a Single-Walled Carbon Nanotube Steffi Friedrichs, Angus I. Kirkland, Rüdiger R. Meyer, Jeremy Sloan, and Malcolm L.H. Green

Searching Ultimate Nanometrology for AlOx Thickness in Magnetic Tunnel Junction by Analytical Electron Microscopy and X-Ray

Reflectometry Se Ahn Song, Tatsumi Hirano, Jong Bong Park, Kazutoshi Kaji, Ki Hong Kim, and Shohei Terada

Damage in III-V Compounds during Focused lon Beam Milling S. Rubanov and P.R. Munroe

Self-Assembled Nanostructures on $\mathrm{VSe}_{2}$ Surfaces Induced by $\mathrm{Cu}$

Deposition

Erdmann Spiecker, Stefan Hollensteiner, Wolfgang Jäger, Hans Haselier, and Herbert Schroeder

Calendar of Meetings and Courses

Indexed in Chemical Abstracts, Current Contents, BIOSIS, and MEDLINE (PubMed)

MSA members receive both Microscopy Today and Microscopy and Microanalysis FREE! 


\section{Group Leader}

Oak Ridge National Laboratory

Ridge National

Laboratory

(ORNL),

nestled in the

ridge and valley

region between

the Great

Smokies and

the Cumberland

Mountains in

East Tennessee,

is a Department

of Energy

multiprogram

science and

technology

laboratory.

Microscopy, Microanalysis,

\section{Microstructures Group}

Metals and Ceramics Division

The Oak Ridge National Laboratory (ORNL) is seeking applications for the position of Leader of the Microscopy, Microanalysis, Microstructures (MMM) Group. The MMM Group houses two world-class user centers - the Shared Research Equipment (SHaRE) User Facility and the Materials Analysis User Center (MAUC) of the ORNL High Temperature Materials Laboratory (HTML), with a concentration in atomic-resolution imaging and microanalysis, and provides diverse microcharacterization facilities to support the largest non-defense materials R\&D effort in the United States. The successful candidate will serve as SHaRE Director and as the ORNL institutional lead in the multi-laboratory Transmission Electron Aberration-corrected Microscope (TEAM) project, as well as provide line management oversight for MAUC, including the JEOL 2200FS Aberration Corrected Electron Microscope (ACEM).

The successful candidate will be a highly qualified scientist with an outstanding research and publication record in technique development and/or applications to materials or condensed matter science in one or more areas of electron microscopy and microanalysis. The responsibilities of the Group Leader include the organization, coordination, and supervision of user center and microscopy support activities, scientific interaction with ORNL colleagues and external users, and the direction of a large and diverse research group of scientific and technical staff members. The position offers an enthusiastic, strongly collaborative and creative research environment, competitive salary, and opportunities for making a significant impact across the entire spectrum of condensed matter and materials sciences. Excellent interpersonal as well as oral and written communication skills are expected.

To apply for this position, candidates should go to the Oak Ridge National Laboratory web-site (www.ornl.gov), click on the "Jobs" category and then "ORNL Employment." Under "Scientific/Tech Staff/Mgmt," look for Group Leader Microscopy, Microanalysis, Microstructures Group.

After officially applying on line, qualified candidates should also submit a CV electronically

by October 17, 2005, to Karen Simonson (simonsonkp@ornl.gov), Metals and Ceramics Division, Oak Ridge National Laboratory.

Additional information about the Oak Ridge National Laboratory is available from http://www.ornl.gov

UT-Battelle is committed to Equal Employment Opportunity for all persons.

ORNL, a multiprogram research facility managed by UT-Battelle, LLC, for the U.S. Department of Energy, is an equal opportunity employer committed to building and maintaining a diverse work force.

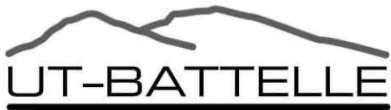

Quality Preparation Quality Results

Advanced EM Specimen Preparation Instuments
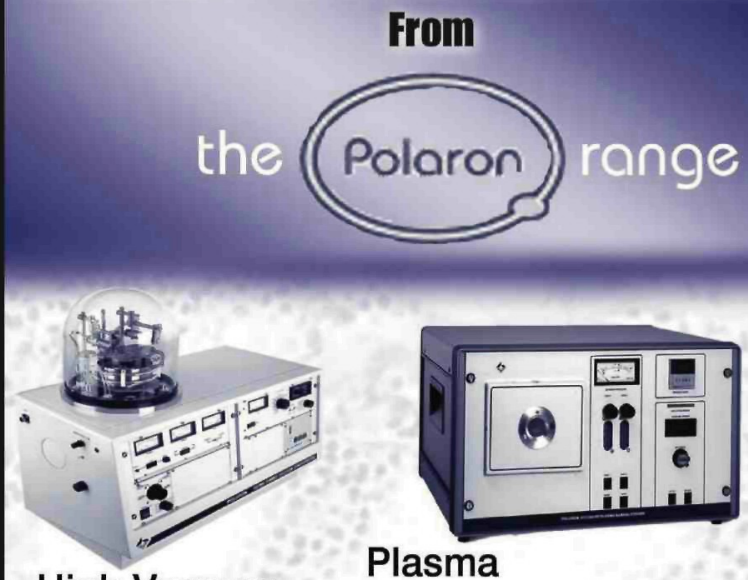

High Vacuum Evaporation

Plasma

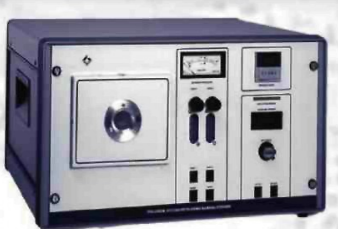

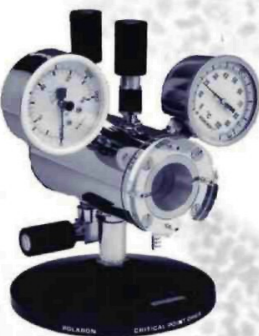

Critical Point Drying

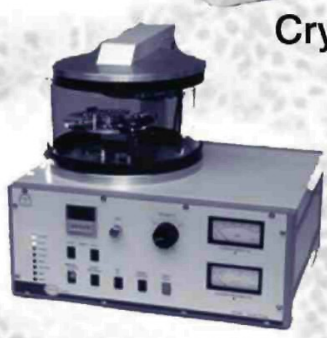

SEM

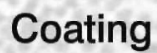

Introilucing the E6500 Evaporator

Low Gost - Full Features - Revolutionary Design

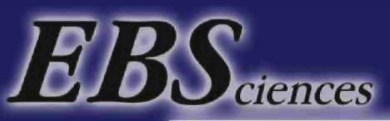

ADDING BRILUANCE TO YOUR VISION email: ebs@ebsciences.com www.ebsciences.com 